\title{
Extreme Levitation of Colloidal Particles in Response to Oscillatory Electric
}

Fields

\section{Authors:}

Scott C. Bukosky, ${ }^{1,2}$ S. M. H. Hashemi Amrei, ${ }^{1}$ Sean P. Rader, ${ }^{1}$ Jeronimo Mora, ${ }^{1}$ Gregory Miller, ${ }^{1, \dagger}$ William D. Ristenpart ${ }^{1, \dot{*}}$

${ }^{1}$ Department of Chemical Engineering, University of California Davis, Davis, California 95616, United States

${ }^{2}$ Lawrence Livermore National Laboratory, Livermore, CA 94551, USA

$\dagger$ Email-grgmiller@ucdavis.edu

+ Correspondence to: Email - wdristenpart@ucdavis.edu

\section{Supplementary Figure}

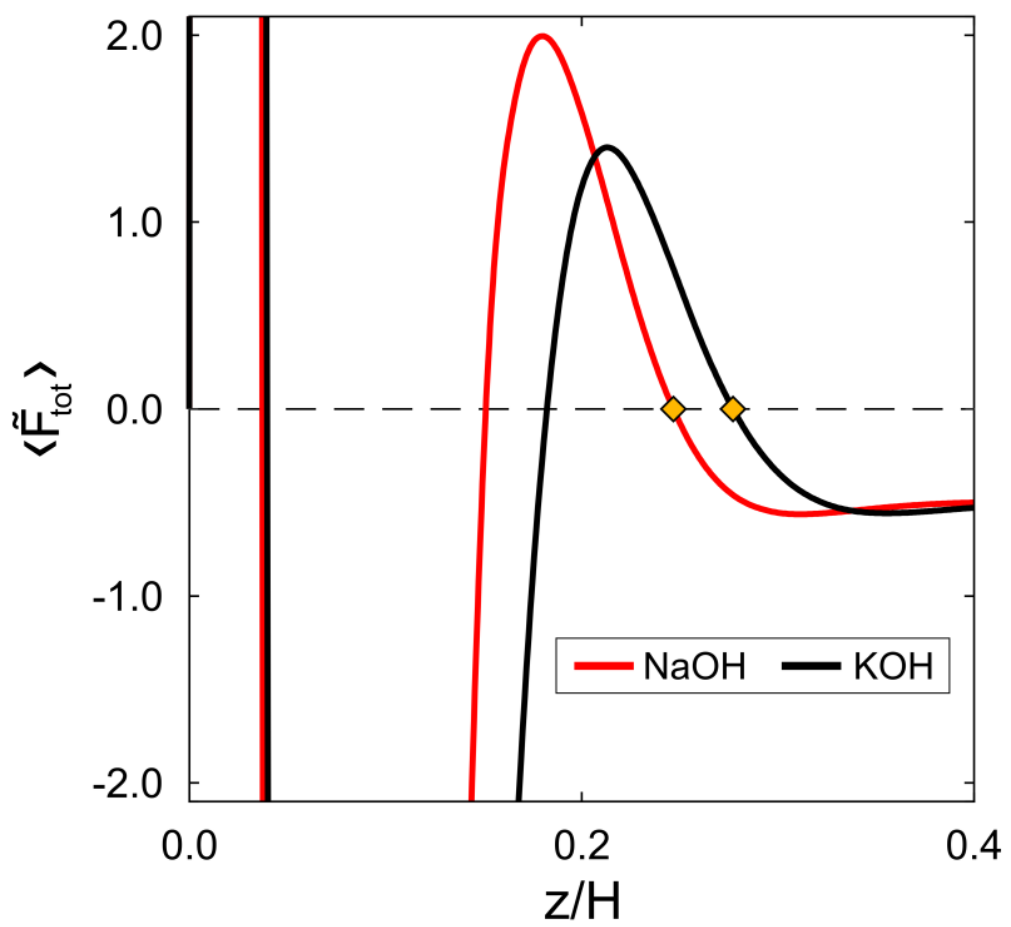

Figure S1. Electrolyte stable point comparison. Predicted AREF-induced force distributions $\left(\left\langle\tilde{F}_{\text {tot }}\right\rangle=\left\langle F_{\text {tot }}\right\rangle a / k_{B} T\right)$ and the corresponding fixed point stability diagrams comparing $\mathrm{NaOH}$ and $\mathrm{KOH}$ electrolyte solutions. Yellow diamond markers show the location of the upper stable fixed points and their dependence on each given variable. Parameters: $f=200 \mathrm{~Hz}, \phi_{o}=30 k_{B} T / e$, $H=50 \mu m, d=2 \mu m, \delta=3.96(\mathrm{NaOH})$ and $2.69(\mathrm{KOH})$. 